\title{
Experimental study of impact properties of aluminium honeycomb sandwich structure
}

\author{
Ondrej Flasar ${ }^{1, *}$, Vaclav Triska ${ }^{1}$, and Milan Junas ${ }^{1}$ \\ ${ }^{1}$ University of Defence, Department of Air Force and Aircraft Technology, Faculty of Military Technology, Kounicova 65, \\ 66210 Brno, Czech Republic
}

\begin{abstract}
Aircraft grade of structural sandwich plates has been studied to determine the influence rate of particular face sheets adjustments on impact energy absorption. The face sheets were fabricated by carbon fibre reinforced plastic (CFRP) laminas using unidirectional reinforcement with either cross-ply [0/90 $]_{\mathrm{n}}$ or angle-ply $[ \pm 45]_{\mathrm{n}}$ orientation of fibres. A core of the sandwich plates was then formed by aluminium honeycomb. The impact properties of small unnotched sandwich specimens were measured by instrumented Charpy impact test device. Assessment of force-displacement curve, loading process and failure mode was carried out for each specimen type to determine a general trend for increasing the impact properties of sandwich structures.
\end{abstract}

\section{Introduction}

Sandwich structures composed of strong skins and a lowdensity core provide very stiff structure units in many applications including aerospace, train industry, maritime etc. Except for their high strength property sandwiches excel at impacts and vibrations absorption. The damage response of sandwich panels depends on the mechanical properties of the core material and the fracture characteristics of the skin [1].

When observing energy absorbing properties, there are a few damage processes which can deal with impact loading such as core buckling, cells or foam folding, delamination, fibre fracture etc. [2-4]. Research can thus focus on either different core materials including foams, honeycomb, cork, balsa wood, coconut and other artificially constructed truss or sheet structures [4-11] or different face sheets as aluminium, carbon, glass, aramid reinforced composites etc. [3, 12]. Most of the studies use drop weight methods for the evaluation of sandwich structures in the range of low-medium velocity impacts $[2,3,8,12,13]$. There can be found also impact test apparatuses such as the pendulum impactor [14]. However, this study prefers the instrumented Charpy pendulum hammer due to the emphasis on deformation behaviour, total absorbing energy and test repeatability.

Since there does not exist general standard or advisory material for generic sandwich beam testing using a pendulum impactor, the only relative standard is ASTM D 6110 [15] concerning notched plastic material testing.

Due to lack of information about the differences in the dynamic behaviour of structural sandwiches with different stacking sequences of their face sheets, this paper focuses on determining the behaviour and trends affected by various adjustments of the tested sandwiches.
For that purpose we used pure CFRP (carbon fibre reinforced plastics) materials and subsequently sandwich plates with face sheets made of CFRP with similar stacking sequences.

\section{Material and method}

\subsection{Test specimen}

Both pure CFRP and sandwich specimens have an unnotched beam shape derived from specimen described in the standard (standard). The CFRP material contains unidirectional reinforcement cured by epoxy resin. The fibre volume fraction of this material is $60 \%$. Two stacking sequences with orientation either cross-ply $[0 / 90]$ or angle-ply $[ \pm 45]$ through the whole thickness were applied. The dimensions of CFRP specimens are as follows: length $l=80 \mathrm{~mm}$, width $w=10 \mathrm{~mm}$ and thickness $t=6 \mathrm{~mm}$.

The sandwich specimens are then laid up by CFRP face sheets and aluminium honeycomb core. The used CFRP sheets with fibre volume fraction $F V=50 \%$ are cross-ply $[0 / 90]_{2}$, angle-ply $[ \pm 45]_{2}$ and thicker angle-ply $[ \pm 45]_{4}$. The aluminium honeycomb density is $32 \mathrm{~kg} / \mathrm{m}^{3}$ and the hexagonal cell has a size of $6,4 \mathrm{~mm}$. The dimensions of the sandwich specimens are the same (illustrated in Fig. 1 ) as those of CFRP specimens except for width which is extended to $w=20 \mathrm{~mm}$. All observed composite or sandwich versions had five tested specimens. The applied impact force is always oriented normal to the specimen plane.

\footnotetext{
${ }^{*}$ Corresponding author: ondrej.flasar@unob.cz
} 


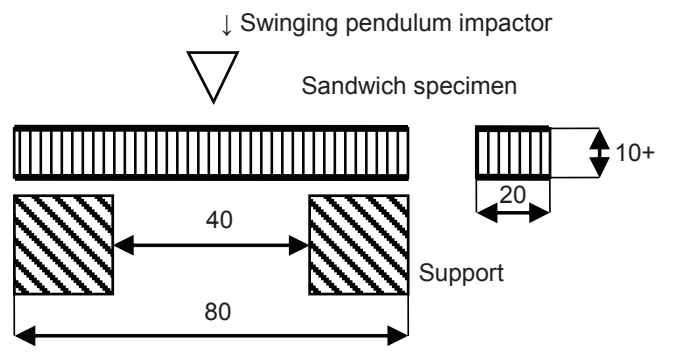

Fig. 1. Specimen dimensions and mutual position of the specimen and testing device.

\subsection{Instrumented impact test}

The device used for impact testing was instrumented Charpy pendulum Zwick-Roell 300. The pendulum hammer reaches a peripheral velocity of 5,234 $\mathrm{ms}^{-1}$ and a kinetic energy of $300 \mathrm{~J}$ at the impact point. The length of the support span is $40 \mathrm{~mm}$. The instrumented pendulum records the whole force-displacement curve which is then used for results assessment. An integration of the curve gives a total value of absorbed energy $W_{t}$ during specimen deformation:

$$
W_{t}=\int_{0}^{s} F s d s,
$$

where $F$ is force and $s$ is standard travel. The differences caused by different face sheet thickness are suppressed by the introduction of a value of absorbed energy per unit area $a_{k}$ (cross-sectional):

$$
a_{k}=\frac{W_{t}}{w t} .
$$

In order to have more unbiased result assessment specific absorbed energy, which is dependent on both cross-section and weight of specimen, is then applied:

$$
a_{m}=\frac{W_{t}}{w t m},
$$

where $m$ is the weight of the specimen. All results, forcedisplacement curves and optical micrograms of specimens after failure were assessed during the post-processing.

\section{Results and discussion}

The results are studied in two phases. The first phase describes results gained during measurement of CFRP specimens. The other phase elaborates on the results of selected sandwich structures with respect to knowledge of pure CFRP behaviour.

Fig. 2 shows results of averaged absorbed impact energy $a_{k}$ by comparing two CFRP stacking sequences. The angle-ply $[ \pm 45]$ laminate exhibits a significantly higher absorbed energy $\left(328,7 \mathrm{kJm}^{-2}\right)$ than the cross-ply [0/90] laminate $\left(221,8 \mathrm{kJm}^{-2}\right)$. Nevertheless, the stiffness and strength of fibres oriented in $0^{\circ}$ direction demonstrate its capability when comparing maximum reached load $F_{m}$ (Fig. 3). In this case, the cross-ply [0/90] laminate shows more than two times higher value of averaged ultimate load $(4557 \mathrm{~N})$ while angle-ply [ \pm 45 ] laminate reached only $2187 \mathrm{~N}$. Accordingly, it should be noted that total absorbed energy is not directly proportional to ultimate load.

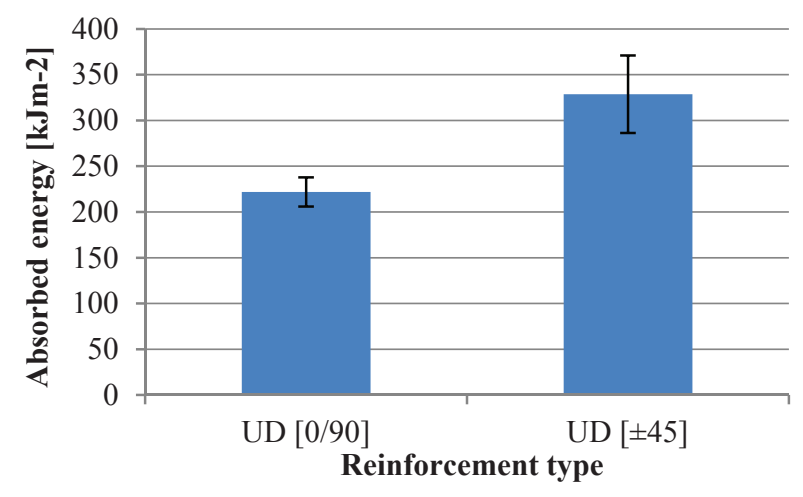

Fig. 2. Specific absorbed impact energy of CFRP specimens, error bars included.

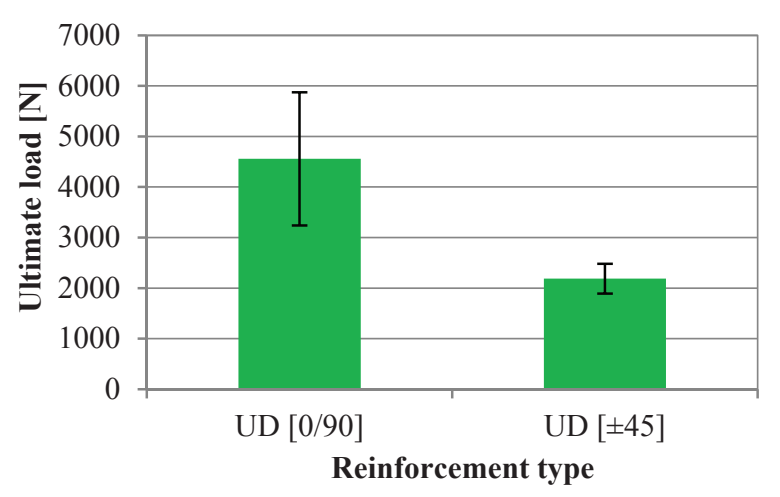

Fig. 3. Ultimate load of CFRP specimens, error bars included.

The fact of the low absorbed energy of cross-ply [0/90] laminate is obvious from Fig. 4. Even if this specimen reaches high load, the overall behaviour is quite vulnerable. The growth of the applied force in the beginning of loading exposes very stiff property. However, after reaching its maximum there is a steep fall to lower values with the very unstable process until the total failure. The sharp peak indicates brittle crack of the several plies on the top. Subsequently, the following unstable deformation characterizes numerous delaminations and matrix cracks at $90^{\circ}$ plies. This process can be described as explosive fraying of the specimen edge.

The other angle-ply $[ \pm 45]$ laminate exhibits rather plastic behaviour. The smooth force-displacement curve shows short lasting on maximum load value and a following gradual decrease to zero value. The transition between elastic deformation and constant phase of the force-displacement curve can be labelled as yield point, which identifies the moment where irreversible deformation occurs. This type of CFRP is characterized by extensive fibre pull out failure which causes its resilient post-failure integrity. This material behaviour can thus be called tough. 
Unidirectional cross-ply $[0 / 90]_{\mathrm{n}}$ laminate $a_{k}=221,8 \mathrm{kJm}^{-2}, F_{m}=4557 \mathrm{~N}$

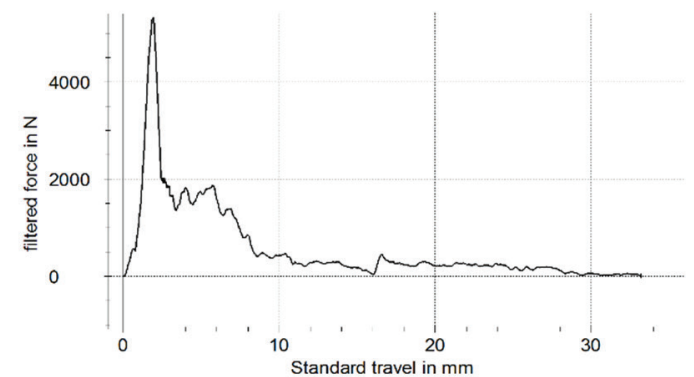

Unidirectional angle-ply $[ \pm 45]_{\mathrm{n}}$ laminate $a_{k}=328,7 \mathrm{kJm}^{-2}, F_{m}=2187 \mathrm{~N}$

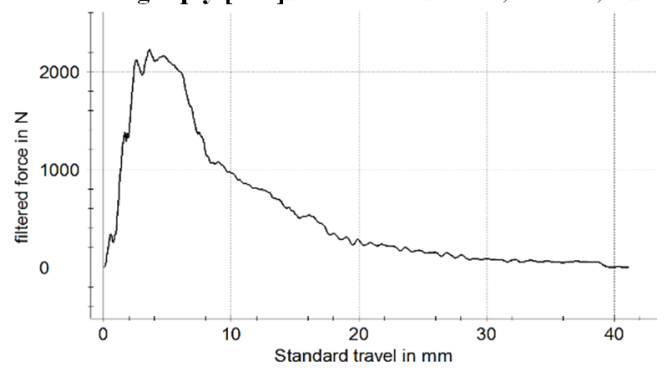

Sandwich with face sheets $[ \pm 45]_{2}, a_{k}=31,1 \mathrm{kJm}^{-2}, F_{m}=281 \mathrm{~N}$

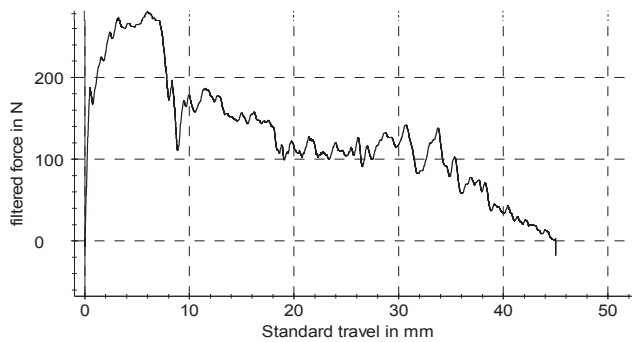

Sandwich with face sheets $[0 / 90]_{2}, a_{k}=33,8 \mathrm{kJm}^{-2}, F_{m}=329 \mathrm{~N}$

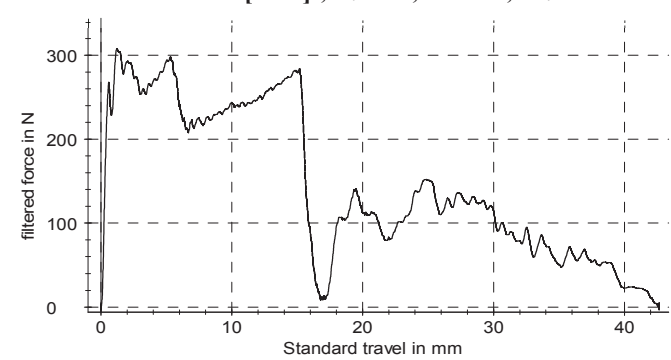

Sandwich with face sheets $[ \pm 45]_{4}, a_{k}=61,1 \mathrm{kJm}^{-2}, F_{m}=580 \mathrm{~N}$

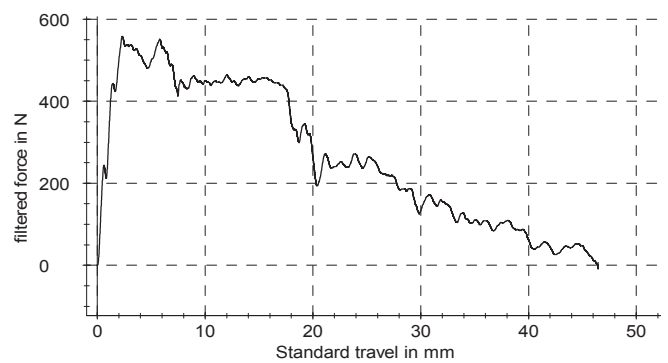

Fig. 4. Results of impact test.
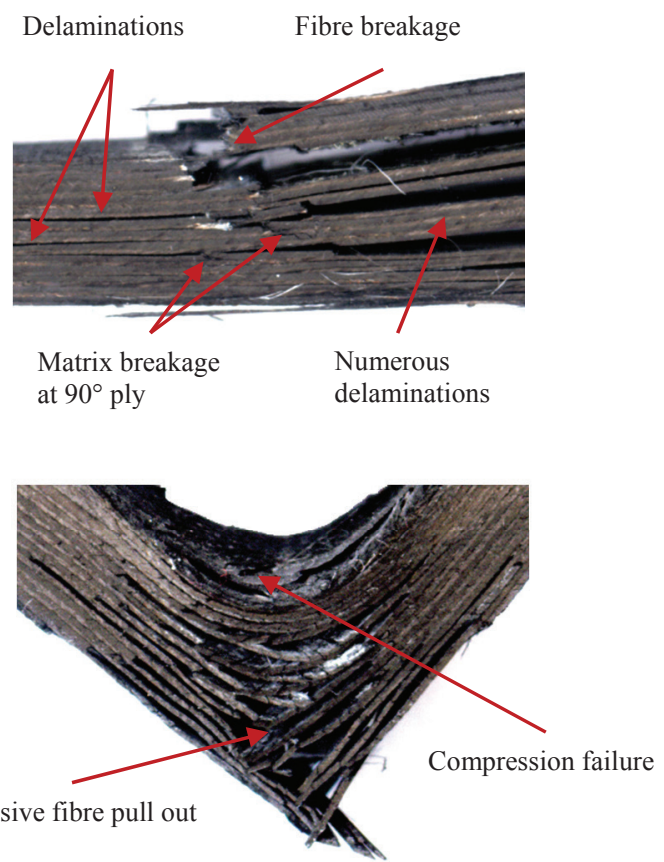

Fibre pull out
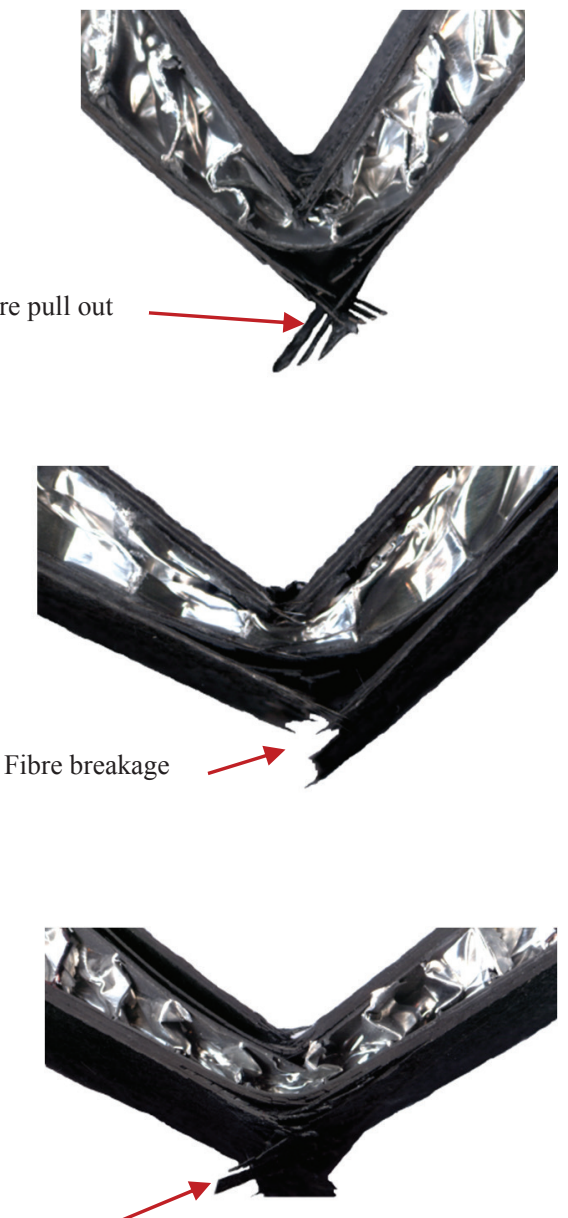

Fibre pull out 
There was hereby established the behaviour of CFRPs with different stacking sequence. The research carries on testing of sandwich panels. Obviously, the sandwich with 8-ply face sheets gains the highest values of absorbed energy $a_{k}$ (Fig. 5). When comparing sandwiches with four plies in the skin, the $[0 / 90]_{2}$ sandwich exhibits slightly better properties than the $[ \pm 45]_{2}$ sandwich. Nevertheless, specific absorbed energy $a_{m}$ (Fig. 6) related to the weight of each specimen provides an interesting comparison. This parameter demonstrates considerable increase of the weight of the thicker sandwich specimen, which means that the total difference is more than $80 \% a_{k}\left(27 \mathrm{kJm}^{-2}\right)$ as compared to 4-ply skin sandwich $[0 / 90]_{2}$, which is reduced to about $7 \% a_{m}\left(468 \mathrm{~kJ} / \mathrm{kg}^{-1} \mathrm{~m}^{-2}\right)$ when taking the weight into account.

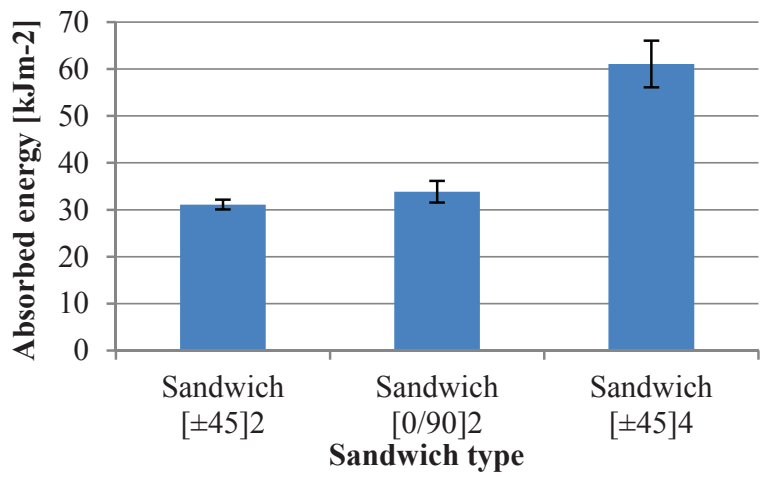

Fig. 5. Specific absorbed impact energy of sandwich specimens, error bars included.

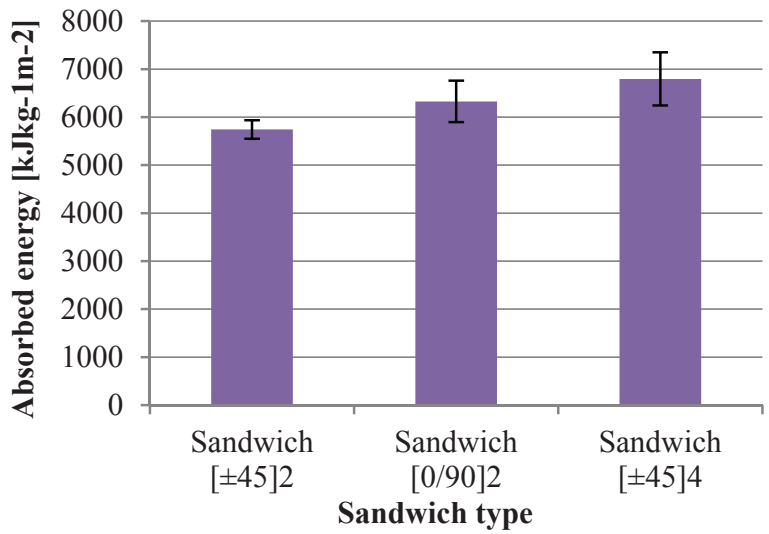

Fig. 6. Specific absorbed impact energy considering the weight of sandwich specimens, error bars included.

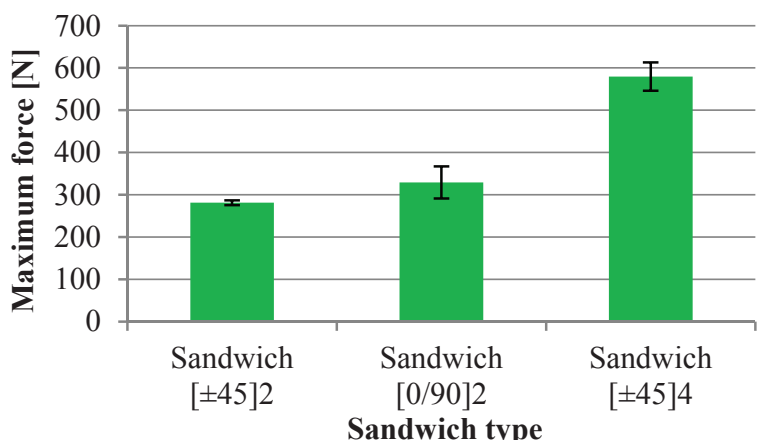

Fig. 7. Ultimate load of sandwich specimens, error bars included.
The $[ \pm 45]_{4}$ sandwich still attains the highest results, but the increase of more than $60 \%$ of the weight (Table 1) makes it less profitable. Next Fig. 7 shows the results of the maximum force reached $F_{m}$. There is an obvious similar trend in value increase as in Fig. 5. Forcedisplacement curves and optical micrograms give a more detailed view of failure behaviour of the tested sandwich specimens again (Fig. 4).

The first sandwich with face sheets $[ \pm 45]_{2}$ exhibits similar behaviour with CFRP $[ \pm 45]$ regarding fibres pulled out of the matrix. The force-displacement curve of the sandwich shows typical bench shape up to deformation $8 \mathrm{~mm}$ on the level of near maximum force. The beginning of bench means loss of stability of cells compressively loaded under impactor, whereas the end of bench characterizes the loss of stability of the whole beam and beginning of plastic bending. The rest of the curve produces a shear folding of honeycomb cells and a skin bending.

Furthermore, the sandwich specimen with face sheets $[0 / 90]_{2}$ demonstrates unstable and vulnerable failure behaviour. On the force-displacement curve it occurs as the first brittle crack of upper skin with compressive deformation of cells characterized by an unstable short bench near force maximum. The gradual compressive folding of cells and elastic bending of lower skin cause the subsequent increase of applied load. However, the brittle crack of the lower skin produces steep decrease to nearly zero values of force. The rest of the following unstable process induces shear folding of cells. When comparing sandwich with $[0 / 90]_{2}$ skin with pure $[0 / 90]$ CFRP, there does not occur explosive delamination but only single delamination due to the low number of plies. The last thicker sandwich specimen $[ \pm 45]_{4}$ exhibits behaviour similar to thinner sandwich $[ \pm 45]_{2}$. Obviously, the whole loading process is moved to higher values of the applied force. The bench shape of the curve on values close to maximum force is extended to deformation 16 - $20 \mathrm{~mm}$ unlike the $[ \pm 45]_{2}$ sandwich. There also occurs an extended region of compressive failure of honeycomb cells. This is caused by thicker face sheet which is able to spread impact force better to the core of the sandwich.

In this way the failure behaviour of different sandwich structures was described. All covered sandwiches achieve conformity in the case of core deformation where compressive deformation occurs first and then shear deformation. The force-displacement curves also reveal the difference between failure behaviour of the sandwiches with $[0 / 90]_{2}$ and $[ \pm 45]_{2}$ face sheets. Even if the sandwich with $[0 / 90]_{2}$ face sheet reached higher values of absorbed energy $a_{k}$ and maximum force $F_{m}$, due to its unstable and brittle behaviour it is less suitable as an energy absorber or a damper.

\section{Conclusion}

An overview of the comparison of the listed pure CFRPs and the sandwiches could state that the behaviour of specimens with a stacking sequence produced in a similar way is analogous. Both sandwiches with skin orientation in $[ \pm 45]$ exhibit a plastic type of force displacement curve 
unlike the sandwich with skin orientation in [0/90], which is combined with brittle traces. Hence, the $[ \pm 45]$ sandwiches and CFRPs are better for application as an energy absorber or a damper.

When comparing sandwiches only, the thickest sandwich with face sheets $[ \pm 45]_{4}$ has gained the highest average of absorbed energy $a_{k}$. However, the weight increase degrades its advantage. The research will thus continue with an analysis of sensitivity on various influences created by changing thickness skin and core thickness using constant materials.

\section{References}

1. S. Abrate, Impact on composite structures. New York: Cambridge University Press, (1998)

2. Y. Shen, F. Yang, W. Cantwell, S. Balawi, Y. $\mathrm{Li}$, Journal of Reinforced Plastics and Composites, 33(12), 1148-1157. DOI: 10.1177/0731684414524465, (2014)

3. A. Hazizan, W. Cantwell, The low velocity impact response of foam-based sandwich structures, DOI: 10.1016/S1359-8368(02)00009-4, (2002)

4. S. Pingle, N. Fleck, V. Deshpande, H. Wadley, Int. J. of Solids and Structures, 48(25-26), 3417-3430, DOI: 10.1016/j.ijsolstr.2011.08.004, (2011)

5. S. Wang, S. Li, J. He, Composite Structures, 163, 312324, DOI: 10.1016/j.compstruct.2016.12.050, (2017)

6. T. George, V. Deshpande, K. Sharp, H. Wadley, Composite Structures, 108, 696-710, DOI: 10.1016/j.compstruct.2013.10.002, (2014)

7. T. Liu, S. Hou, X. Nguyen, X. Han, Composites Part B: Engineering, 114, 328-338, DOI: 10.1016/j.compositesb.2017.01.035, (2017)

8. H. Wang, K. Ramakrishnan, K. Shankar, Materials and Design, 99, 68-82, DOI: doi.org/10.1016/j.matdes.2016.03.048, (2016)

9. G. Sun, S. Li, Q. Liu, G. Li, Q. Li, Composite Structures, 152, 969-993. DOI: 10.1016/j.compstruct.2016.06.019, (2016)

10. G. Kooistra, H. Wadley, Materials, 28(2), 507-514. DOI: 10.1016/j.matdes.2005.08.013. ISSN 02613069, (2007)

11. R. Mines, S. Tsopanos, Y. Shen, R. Hasan, S. Mckown, Int. J. of Impact Engineering, 60, 120-132, DOI: 10.1016/j.ijimpeng.2013.04.007, (2013)

12. V. Birman, Composites Part B: Engineering, 35(6-8), 665-672, DOI: 10.1016/j.compositesb.2003.08.012, (2004)

13. M. Meo, R. Vignjevic, G. Marengo, Int. J. of Mechanical Sciences. 47(9), 1301-1325, DOI: 10.1016/j.ijmecsci.2005.05.006, (2005)

14. D. Zhang, Q. Fei, P. Zhang, Composite Structures, $\quad \mathbf{1 6 8}, \quad 633-645, \quad$ DOI: 10.1016/j.compstruct.2017.02.053, (2017)

15. ASTM D6110-10, Standard Test Method for Determining the Charpy Impact Resistance of
Notched Specimens of Plastics, ASTM International, West Conshohocken, PA, www.astm.org, (2010) 